\title{
RISK MEASURES AND MULTIVARIATE EXTENSIONS OF BREIMAN'S THEOREM
}

\author{
ANNE-LAURE FOUGERES *** AND \\ CECILE MERCADIER, ${ }^{* * * *}$ Université Lyon 1
}

\begin{abstract}
The modeling of insurance risks has received an increasing amount of attention because of solvency capital requirements. The ruin probability has become a standard risk measure to assess regulatory capital. In this paper we focus on discrete-time models for the finite time horizon. Several results are available in the literature to calibrate the ruin probability by means of the sum of the tail probabilities of individual claim amounts. The aim of this work is to obtain asymptotics for such probabilities under multivariate regular variation and, more precisely, to derive them from extensions of Breiman's theorem. We thus present new situations where the ruin probability admits computable equivalents. We also derive asymptotics for the value at risk.
\end{abstract}

Keywords: Discrete-time model; ruin probability; value at risk; multivariate regular variation; dependent risk

2010 Mathematics Subject Classification: Primary 91B30

Secondary 62P05

\section{Introduction}

Let $x$ be a nonnegative real number, let $\left(\rho_{i}\right)_{i \geq 1}$ and $\left(X_{i}\right)_{i \geq 1}$ be two sequences of random variables (RVs), and define the sequence $\left(R_{i}\right)_{i \in \mathbb{N}}$ by the recursive equation

$$
R_{0}=x, \quad R_{i}=R_{i-1}\left(1+\rho_{i}\right)-X_{i}, \quad i \geq 1 .
$$

This model has been used to model insurance risks. In such a context, $x$ denotes the initial capital, $\rho_{i} \in(-1, \infty)$ denotes the random interest rate for the $i$ th period, and $X_{i}$ denotes the total claim amount minus the total premium income, so that $R_{i}$ represents the discounted surplus computed from period 0 to $i$. In this paper we focus on discrete-time models for a fixed finite time horizon $d$.

The ruin probability has become a standard risk measure to assess regulatory capital in insurance. For model (1), the ruin probability within the finite time horizon $[0, d]$ and initial capital reserve $x$ is defined by

$$
\psi(x, d)=\mathrm{P}\left(\min _{1 \leq k \leq d} R_{k}<0 \mid R_{0}=x\right) .
$$

Introducing the so-called discount factor from period $i$ to 0 , denoted by $Y_{i}=\prod_{j=1}^{i}\left(1+\rho_{j}\right)^{-1}$,

Received 25 January 2011; revision received 16 December 2011.

* Postal address: Université de Lyon, CNRS UMR 5208, Université Lyon 1, Institut Camille Jordan, 43 Boulevard du 11 Novembre 1918, F-69622 Villeurbanne cedex, France.

** Email address: fougeres@math.univ-lyon1.fr

*** Email address: mercadier@math.univ-lyon1.fr 
we can re-express the discrete-time risk model as

$$
R_{0}=x, \quad R_{i}=Y_{i}^{-1}\left(x-\sum_{j=1}^{i} X_{j} Y_{j}\right), \quad i \geq 1 .
$$

Hence, the ruin probability can be written as

$$
\psi(x, d)=\mathrm{P}\left(\max _{1 \leq k \leq d} \sum_{i=1}^{k} X_{i} Y_{i}>x\right) .
$$

Several results exist on the limiting behavior of $\psi(x, d)$, especially in the case of an initial reserve $x$ tending to $\infty$, and when the distribution function of the $X_{i} \mathrm{~s}$ is subexponential and sometimes heavy tailed. Most of the existing results are stated under independence between the components of $\boldsymbol{X}=\left(X_{1}, \ldots, X_{d}\right)$. For $\boldsymbol{Y}=\left(Y_{1}, \ldots, Y_{d}\right)$ deterministic, these asymptotics are given in Embrechts and Veraverbeke (1982), Sgibnev (1996), Embrechts et al. (1997, Chapter 1), $\mathrm{Ng}$ et al. (2002), and Zhu and Gao (2008), among others. The case where the $Y_{i}$ s are bounded RVs has been studied in Tang and Tsitsiashvili (2003a), (2003b). Models governed by a specific dependence structure of the random vector $\boldsymbol{Y}$ have been proposed in Nyrhinen (1999), Cai (2002), and Chen and Su (2006). Recent extensions to any dependence structure of $\boldsymbol{Y}$ can be found in Goovaerts et al. (2005), Wang et al. (2005), and Wang and Tang (2006), where the results are proven under a moment condition.

In some situations, the assumption of independence between the claim amounts $X_{i}$ might be unrealistic. Cossette and Marceau (2000) considered special models of dependency. Allowing general dependence among the $X_{i}$ s but keeping them independent from the nonnegative weights $Y_{i}$, Zhang et al. (2009) derived approximations for $\psi(x, d)$ under the assumptions that the $X_{i} \mathrm{~s}$ have (extended) regularly varying tail and are asymptotically independent. Roughly speaking, the latter notion means that the joint upper tail of two claim amounts is negligible compared with each univariate tail. More precisely, Zhang et al. (2009) established the following equivalences, as $x$ tends to $\infty$ :

$$
\psi(x, d) \sim \mathrm{P}\left(\sum_{i=1}^{d} X_{i} Y_{i}>x\right) \sim \sum_{i=1}^{d} \mathrm{P}\left(X_{i} Y_{i}>x\right) .
$$

In their context, the first equivalence in (2) is a consequence of the assumption that the left tails of the $X_{i}$ s are lighter than their right tails (see (6) below for a precise definition) and of the positivity of the weights $Y_{i}$. The second equivalence in (2), which follows from the asymptotic independence of the $X_{i} \mathrm{~s}$, is also of interest in its own right. Indeed, several papers are concerned with how the tail of the marginal distribution of an individual summand influences the asymptotic behavior of the sum. Barbe et al. (2006) extended the equivalence between the tail of a sum and the sum of the tails, already obtained in Wüthrich (2003) and Alink et al. (2004), (2005), to a broader class of dependence structures using multivariate extreme value theory. Recently, Kortschak and Albrecher (2009) treated the case of nonidentically distributed and not necessarily positive RVs, and Foss and Richards (2010) obtained results for sums of conditionally independent subexponential RVs.

The aim of this paper is to establish, in various contexts of dependence, asymptotics of the ruin probability and the value at risk. Our generalizations of (2) are in two directions: we allow dependence between the claim amounts $X_{i}$ and the weights $Y_{i}$; and we relax the assumption 
of asymptotic independence of the claim amounts. These results are obtained under the key assumption that $\boldsymbol{X}$ is multivariate regularly varying at infinity; see, for instance, Resnick (2007, Chapter 6). More precisely, we highlight the fact that our extensions are valid as soon as the random vector $\left(X_{1} Y_{1}, \ldots, X_{d} Y_{d}\right)$ is also multivariate regularly varying, which is known as a Breiman-type result.

The rest of the paper is organized as follows. Section 2 contains the notation and some preliminary results. Several generalizations of Breiman's theorem are stated in Section 3. In Section 4 we take advantage of these extensions to derive asymptotics for the ruin probability and the value at risk. A brief conclusion is given in Section 5. The proofs are postponed until Section 6.

\section{Notation, definitions, and preliminary results}

We start this section with some notation. Let $\boldsymbol{x} \cdot \boldsymbol{y}$ define the componentwise product of two vectors $\boldsymbol{x}=\left(x_{1}, \ldots, x_{d}\right)^{\top}$ and $\boldsymbol{y}=\left(y_{1}, \ldots, y_{d}\right)^{\top} \in \mathbb{R}^{d}$, i.e.

$$
\boldsymbol{x} \cdot \boldsymbol{y}=\left(x_{1} y_{1}, \ldots, x_{d} y_{d}\right)^{\top}
$$

and let

$$
z^{-1}=\left(z_{1}^{-1}, \ldots, z_{d}^{-1}\right)^{\top}
$$

be the componentwise inverse of $z \in[0, \infty]^{d}$. We define the inverse image of a set $A$ for $\boldsymbol{y} \in[0, \infty]^{d}$ by

$$
\boldsymbol{y}^{-1} \cdot A=\left\{\boldsymbol{x} \in \mathbb{R}^{d} \mid \boldsymbol{y} \cdot \boldsymbol{x} \in A\right\}=\left\{\left(y_{1}^{-1} x_{1}, \ldots, y_{d}^{-1} x_{d}\right)^{\top}, \boldsymbol{x} \in A\right\} .
$$

Let $\boldsymbol{X}$ be a $\boldsymbol{d}$-dimensional random vector. We say that $\boldsymbol{X}$ is multivariate regularly varying (MRV) if there exists a nonnull Radon measure $v$ on $\mathscr{B}_{d}$, the Borel $\sigma$-field of $\mathbb{E}_{d}=[-\infty, \infty]^{d} \backslash\{\mathbf{0}\}$, such that $v\left((-\infty, \infty)^{d} \backslash\{\boldsymbol{0}\}\right)>0$ and a normalizing function $a$ (with $a(t) \rightarrow \infty$ ) such that

$$
t \mathrm{P}\left(\frac{X}{a(t)} \in \cdot\right) \stackrel{\mathrm{v}}{\rightarrow} v
$$

as $t$ tends to $\infty$, where $\stackrel{\mathrm{v}}{\rightarrow}$ ' refers to vague convergence on $\mathcal{B}_{d}$. Recall that a Radon measure on $\mathcal{B}_{d}$ is a measure that is finite on each compact set of $\mathbb{E}_{d}$, and that a set $A \subset \mathbb{E}_{d}$ is relatively compact if it is bounded away from 0 . Recall also that a sequence of measures $v_{n}$ converges vaguely to $v$ on $\mathscr{B}_{d}$ if $\int_{\mathbb{E}_{d}} f \mathrm{~d} v_{n}$ converges to $\int_{\mathbb{E}_{d}} f \mathrm{~d} v$ for any continuous function $f$ compactly supported on $\mathbb{E}_{d}$, or, equivalently, if $v_{n}(K)$ converges to $v(K)$ for each relatively compact set $K$ of $\mathbb{E}_{d}$ such that $v(\partial K)=0$.

The limit measure $v$ is necessarily homogeneous, i.e. $v(t K)=t^{-\alpha} v(K)$ for some $\alpha>0$ and any relatively compact Borel set $K$ of $\mathbb{E}_{d}$. The function $a$ is regularly varying with index $1 / \alpha$. We will write $\boldsymbol{X} \in \operatorname{MRV}(\alpha, a, v)$ if (3) holds or simply MRV when no confusion is possible.

In convergence (3) we may choose the normalizing function $a$ such that

$$
\lim _{t \rightarrow \infty} t \mathrm{P}(\|\boldsymbol{X}\|>a(t))=1
$$

where $\|\cdot\|$ denotes some norm on $\mathbb{R}^{d}$. Given the choice of a norm on $\mathbb{R}^{d}$, a polar representation of the measure $v$ can be obtained. This result is due to De Haan and Resnick (1977); see also, for instance, Resnick (2007, Theorem 6.1, p. 173 and Section 6.5.5, p. 201). Let $\mathbb{S}_{\|\cdot\|}^{d-1}$ denote 
the unit sphere of $\mathbb{R}^{d}$ relatively to the norm $\|\cdot\|$. Then $X$ is MRV if and only if there exist a measure $H_{\|\cdot\|}(\cdot)$ on $\mathbb{S}_{\|\cdot\|}^{d-1}$, a positive real $\alpha$, and a normalizing function $a$ (with $a(t) \rightarrow \infty$ ) such that

$$
t \mathrm{P}\left(\left(\frac{\|\boldsymbol{X}\|}{a(t)}, \frac{\boldsymbol{X}}{\|\boldsymbol{X}\|}\right) \in \cdot\right) \stackrel{\mathrm{v}}{\rightarrow}\left(\eta_{\alpha} \times H_{\|\cdot\|}\right)(\cdot)
$$

as $t$ tends to $\infty$, where vague convergence holds on $(0, \infty] \times \mathbb{S}_{\|\cdot\|}^{d-1}$ and $\eta_{\alpha}$ denotes the Radon measure on $(0, \infty]$ defined by $\eta_{\alpha}(x, \infty]=x^{-\alpha}$. The measure $H_{\|\cdot\|}$ is called the spectral measure, or angular measure, and the choice of the normalizing function in (4) implies that $H_{\|\cdot\|}$ is a probability measure on $\mathbb{S}_{\|\cdot\|}^{d-1}$. The link between the limit measure $v$ and the spectral measure $H_{\|\cdot\|}$ can be made explicit via the following decomposition. For any $A \in \mathcal{B}_{d}$,

$$
v(A)=\int_{T(A)} \frac{\alpha \mathrm{d} r}{r^{\alpha+1}} H_{\|\cdot\|}(\mathrm{d} w),
$$

where $T(z)=(\|z\|, z /\|z\|)$ for any $z \in \mathbb{E}_{d}$ and $T(A)$ is the usual image of $A$ by $T$.

We will use the following property in the sequel, and sketch the proof in Section 5.

Lemma 1. If $\boldsymbol{X}$ is $\operatorname{MRV}(\alpha, a, v)$ on $\mathbb{R}^{d}$ and if the left tail of each component $X_{i}$ is lighter than its right tail, i.e.

$$
\lim _{x \rightarrow \infty} \frac{\mathrm{P}\left(X_{i}<-x\right)}{\mathrm{P}\left(X_{i}>x\right)}=0, \quad 1 \leq i \leq d,
$$

then the measure $v$ is concentrated on $[0, \infty]^{d} \backslash\{\mathbf{0}\}$, the spectral measure $H_{\|\cdot\|}$ of $\boldsymbol{X}$ is concentrated on $\mathbb{S}_{\|\cdot\|}^{d-1} \cap[0, \infty]^{d}$, and

$$
\lim _{x \rightarrow \infty} \frac{\mathrm{P}\left(\max _{1 \leq j \leq d} \sum_{i=1}^{j} X_{i}>x\right)}{\mathrm{P}\left(\sum_{i=1}^{d} X_{i}>x\right)}=\lim _{x \rightarrow \infty} \frac{\mathrm{P}\left(\sum_{i=1}^{d} X_{i}^{+}>x\right)}{\mathrm{P}\left(\sum_{i=1}^{d} X_{i}>x\right)}=1 .
$$

Remark 1. Following Kortschak and Albrecher (2009, Lemma 3.1), condition (6) may be replaced by

$$
\mathrm{P}\left(X_{i}>a, X_{j}>b\right) \geq \mathrm{P}\left(X_{i}>a\right) \mathrm{P}\left(X_{j}>b\right) \quad \text { for all }(a, b) \in \mathbb{R}^{2} \text { and } 1 \leq i<j<n .
$$

Throughout this paper, we will choose the $\ell_{1}$-norm $\|\cdot\|_{1}$ defined by

$$
\|\boldsymbol{x}\|_{1}=\sum_{j=1}^{d}\left|x_{i}\right|
$$

and we will denote by $\mathbb{S}_{1}^{d-1}$ and $H_{1}$ the corresponding unit sphere and spectral measure, respectively. The motivation for these choices of normalizing function and norm on $\mathbb{R}^{d}$ is that if $\boldsymbol{X}$ is MRV and satisfies the assumptions of Lemma 1, then we have, for all $x>0$,

$$
\lim _{t \rightarrow \infty} t \mathrm{P}\left(\sum_{i=1}^{d} X_{i}>a(t) x\right)=x^{-\alpha} .
$$

For any $d$-dimensional vector $\boldsymbol{X}$, let $Q(\boldsymbol{X})$ denote the limit, when it exists:

$$
Q(X)=\lim _{x \rightarrow \infty} \frac{\mathrm{P}\left(\sum_{i=1}^{d} X_{i}>x\right)}{\sum_{j=1}^{d} \mathrm{P}\left(X_{j}>x\right)}
$$


Similar limits to $Q(\boldsymbol{X})$ have been considered in the literature. The following result can be found in several references and under different assumptions. We refer the reader to, for instance, Alink et al. (2004), Barbe et al. (2006), Kortschak and Albrecher (2009), and Embrechts et al. (2009a), (2009b). The unique assumption needed is multivariate regular variation, as proven in Section 6. The components of a regularly varying random vector are called asymptotically independent if its spectral measure (with respect to any norm) is concentrated on the axes.

Lemma 2. If $\boldsymbol{X}$ is a d-dimensional MRV random vector with index $\alpha>0$ such that (6) holds then $Q(\boldsymbol{X})$ exists and

$$
d^{-(1-\alpha)_{+}} \leq Q(\boldsymbol{X}) \leq d^{(\alpha-1)_{+}} .
$$

If the components of $\boldsymbol{X}$ are asymptotically independent then $Q(\boldsymbol{X})=1$.

Remark 2. Note that these bounds are universal and (obviously) do not depend on any particular choice of norm on $\mathbb{R}^{d}$, even though the proof we give below makes use of the $\ell_{1}$-norm. For analogous comments on the choice of norm, we refer the reader to the remark in Mainik and Rüschendorf (2009) after Equation (13) therein or to Embrechts et al. (2009a, Proposition 4.6).

Remark 3. Note that contrary to Barbe et al. (2006) and Embrechts et al. (2009a, Corollary 4.2), we do not assume that the random variables $X_{i}$ are positive or that they have the same marginal distribution. This implies in particular that the usual standardization $\int w_{i} H_{1}(\mathrm{~d} w)=$ 1/d (see Barbe et al. (2006, Equation (11)) or Beirlant et al. (2004, p. 260)) does not hold here, but it is not needed. Kortschak and Albrecher (2009) also considered such generalizations to nonpositive or nonidentically distributed marginals. However, the bounds given in Lemma 2 are new in this context.

Remark 4. If $\boldsymbol{X}$ is MRV with index $\alpha>0$, for any fixed norm on $\mathbb{R}^{d}, Q(\boldsymbol{X})$ depends only on $\alpha$ and on the associated spectral measure $H$. Thus, with an abuse of notation, we define $Q(\boldsymbol{X})=Q(\alpha, H)$. The properties of $Q(\alpha, H)$ were first investigated in Barbe et al. (2006, Proposition 2.2). For a given $\alpha$, the upper and lower bounds in (7) are achieved by independent or fully dependent components, and, for a given spectral measure $H, Q(\alpha, H)$ is increasing in $\alpha$. See also Kortschak and Albrecher (2009) and Embrechts et al. (2009a), (2009b).

\section{Multivariate extensions of Breiman's theorem}

Let $\boldsymbol{X}$ and $\boldsymbol{Y}$ be two random vectors in $\mathbb{R}^{d}$. A Breiman-type result consists in obtaining sufficient conditions for the vector $\boldsymbol{Y} \cdot \boldsymbol{X}$ to be MRV. We start by recalling a particular case of Basrak et al. (2002, Proposition A.1), who proved the multivariate regular variation of $\boldsymbol{M} \boldsymbol{X}$, where $\boldsymbol{M}$ is a random matrix independent of $\boldsymbol{X}$. Let $v_{\boldsymbol{Y}}$ denote the measure defined on $\mathbb{E}_{d}$ by

$$
v_{\boldsymbol{Y}}(K)=\mathrm{E}\left[v\left(\boldsymbol{Y}^{-1} \cdot K\right)\right]=\int_{[-\infty, \infty]^{d}} v\left(\boldsymbol{y}^{-1} \cdot K\right) \mathrm{P}_{\boldsymbol{Y}}(\mathrm{d} \boldsymbol{y}) .
$$

Theorem 1. Let $\boldsymbol{X} \in \operatorname{MRV}(\alpha, a, v)$. Let $\boldsymbol{Y}$ be a random vector independent of $\boldsymbol{X}$. Assume that there exists a positive $\varepsilon$ such that $0<\mathrm{E}\left[\left|Y_{i}\right|^{\alpha+\varepsilon}\right]<\infty$ for each $i$ in $\{1, \ldots, d\}$. Then the random vector $\boldsymbol{Y} \cdot \boldsymbol{X} \in \operatorname{MRV}\left(\alpha, a, v_{\boldsymbol{Y}}\right)$, i.e.

$$
t \mathrm{P}\left(\boldsymbol{Y} \cdot \boldsymbol{X} \in a(t) \cdot \stackrel{\mathrm{v}}{\rightarrow} v_{\boldsymbol{Y}}\right.
$$

as $t$ tends to $\infty$. 
The hypothesis of independence between $\boldsymbol{X}$ and $\boldsymbol{Y}$ might be restrictive. In the sequel we generalize Theorem 1 with respect to this point. The rest of the section is divided into subsections according to the type of dependence considered between $\boldsymbol{X}$ and $\boldsymbol{Y}$. We investigate three situations that seem meaningful in an actuarial context:

- $\boldsymbol{X}$ is MRV and asymptotically independent of $\boldsymbol{Y}$;

- $\boldsymbol{X}$ has independent and identically distributed (i.i.d.) regularly varying components, and $\boldsymbol{Y}$ is predictable with respect to $\boldsymbol{X}$;

- $\boldsymbol{X}$ and $\boldsymbol{Y}$ are jointly MRV and asymptotically dependent.

In each of these situations, an extension of Breiman's theorem is stated.

\subsection{Case of asymptotic independence}

The first generalization of Theorem 1 is done under the condition that $\boldsymbol{X}$ and $\boldsymbol{Y}$ are asymptotically independent in the following sense. We assume that

$$
t \mathrm{P}\left(\left(\frac{X}{a(t)}, Y\right) \in \cdot\right) \stackrel{\mathrm{v}}{\rightarrow}(v \times L)(\cdot)
$$

on the Borel sets of $\mathbb{E}_{d} \times[-\infty, \infty]^{d}$, where $v$ is a Radon measure on $\mathbb{E}_{d}$ not concentrated at $\infty$ and $L$ is a probability measure on $[-\infty, \infty]^{d}$. Note that the roles of $\boldsymbol{X}$ and $\boldsymbol{Y}$ are not symmetric in this definition since it specifies their independence when $\boldsymbol{X}$ is large only. Condition (8) implies in particular that $\boldsymbol{X} \in \operatorname{MRV}(\alpha, a, v)$ for some positive $\alpha$. This case obviously contains the case of stochastic independence between $X$ and $Y, L$ being then the distribution of $\boldsymbol{Y}$.

Following Maulik et al. (2002), we make the following asymptotic negligibility assumption. Assume that, for some $\delta>0$ and any $i$ in $\{1, \ldots, d\}$,

$$
\lim _{\varepsilon \rightarrow 0} \limsup _{t \rightarrow \infty} t \mathrm{E}\left[\left(\frac{|\boldsymbol{X}|\left|Y_{i}\right|}{a(t)}\right)^{\delta} \mathbf{1}_{\{|\boldsymbol{X}| / a(t) \leq \varepsilon\}}\right]=0
$$

and also that

$$
\int_{[-\infty, \infty]^{d}}\|\boldsymbol{y}\|^{\alpha} L(\mathrm{~d} \boldsymbol{y})<\infty
$$

Let $v_{L}$ be the measure defined on $\mathbb{E}_{d}$ by

$$
v_{L}(A)=(v \times L)\left(\left\{(\boldsymbol{x}, \boldsymbol{y}), \boldsymbol{x} \in \boldsymbol{y}^{-1} \cdot A\right\}\right)=\int_{[-\infty, \infty]^{d}} v\left(\boldsymbol{y}^{-1} \cdot A\right) L(\mathrm{~d} \boldsymbol{y}) .
$$

Note that if $\boldsymbol{Y}^{*}$ is a random vector with distribution $L$ then $v_{L}=v_{\boldsymbol{Y}^{*}}$.

Theorem 2. Suppose that assumptions (8), (9), and (10) hold. Then the random vector $\boldsymbol{Y} \cdot \boldsymbol{X} \in$ $\operatorname{MRV}\left(\alpha, a, v_{L}\right)$, i.e.

$$
t \mathrm{P}\left(\boldsymbol{Y} \cdot \boldsymbol{X} \in a(t) \cdot \stackrel{\mathrm{v}}{\rightarrow} v_{L}\right.
$$

as $t$ tends to $\infty$.

The proofs of Theorem 2 and subsequent results are postponed until Section 6.1. We establish a more general result on a random vector $\boldsymbol{M} \boldsymbol{X}$ in the proof of Theorem 2, where $\boldsymbol{M}$ is a random matrix of size $q \times d$. 


\subsection{Case of predictable weights}

The following generalization is a straightforward consequence of the predictable framework introduced in Hult and Samorodnitsky (2008). In this context, we assume that the components of $\boldsymbol{X}$ are i.i.d. and regularly varying with index $\alpha$ at $\infty$ so that $\boldsymbol{X}$ is $\operatorname{MRV}(\alpha, a, v)$. More precisely, the sequence $t \mathrm{P}(\boldsymbol{X} / a(t) \in \cdot)$ converges vaguely to a measure $v$, which is concentrated on the axes

$$
v=\sum_{j=1}^{d} v_{j},
$$

where $v_{j}(A)=v\left(A \cap \delta_{j}\right)$ and $\delta_{j}=\left\{z \in \mathbb{R}^{d} \mid z_{j} \neq 0, z_{i}=0, i \neq j\right\}$ is the $j$ th punctured coordinate axis.

The predictable framework of Hult and Samorodnitsky (2008) consists of assuming that there exists a filtration with respect to which the $X_{j}$ s are measurable and the $Y_{j}$ s are predictable. This implies in particular that, for each $j, X_{j}$ and $Y_{j}$ are independent.

This framework is of interest in time series. An example is the EGARCH process of Nelson (1991). This process, say $\left\{\zeta_{j}\right\}$, can be expressed as

$$
\zeta_{j}=X_{j} Y_{j}, \quad Y_{j}=\exp \left\{\sum_{i=1}^{\infty} c_{i} \eta_{j-i}\right\},
$$

and the relevant filtration is $\mathcal{F}_{j}=\sigma\left(X_{i}, \eta_{i}, i \leq j-1\right),\left\{X_{j}\right\}$ and $\left\{\eta_{j}\right\}$ are two i.i.d. sequences, not necessarily independent of each other, $\mathrm{E}\left[\eta_{j}\right]=0, \operatorname{var}\left(\eta_{j}\right)=1$, and $\sum_{i=1}^{\infty} c_{i}^{2}<\infty$. The process $\left\{Y_{j}\right\}$ is called the volatility. The extremal properties of this process have been studied in Davis and Mikosch (2001) in the case of independence between the processes $\left\{X_{j}\right\}$ and $\left\{Y_{j}\right\}$.

Theorem 3. Let $\boldsymbol{X}$ and $\boldsymbol{Y}$ be two random vectors of $\mathbb{R}^{d}$. Assume that the components of $\boldsymbol{X}$ are i.i.d. and regularly varying with index $\alpha$ at $\infty$ so that $\boldsymbol{X}$ is $\operatorname{MRV}(\alpha, a, v)$. Suppose that there exists a filtration $\left\{\mathcal{F}_{j}\right\}$ such that $X_{j}$ is $\mathcal{F}_{j+1}$-measurable and $Y_{j}$ is $\mathcal{F}_{j}$-measurable. Assume moreover that there exists $\varepsilon>0$ such that

$$
\mathrm{E}\left[\left|Y_{j}\right|^{\alpha+\varepsilon}\right]<\infty \text { for all } j=1, \ldots, d .
$$

Then the random vector $\boldsymbol{Y} \cdot \boldsymbol{X}$ is MRV and

$$
t \mathrm{P}(\boldsymbol{Y} \cdot \boldsymbol{X} \in a(t) \cdot) \stackrel{\mathrm{v}}{\rightarrow} \sum_{j=1}^{d} \mathrm{E}\left[\left|Y_{j}\right|^{\alpha}\right] v_{j}
$$

as $t$ tends to $\infty$.

\subsection{Case of joint multivariate regular variation}

When neither independence nor asymptotic independence is a relevant assumption, one might be interested in extensions of Breiman's theorem under asymptotic dependence. Specifically, we assume that $\boldsymbol{X}$ and $\boldsymbol{Y}$ are jointly MRV, i.e. there exist $a$ and $b$ such that

$$
t \mathrm{P}\left(\left(\frac{X_{i}}{a(t)}, \frac{Y_{i}}{b(t)}\right)_{i=1, \ldots, d} \in \cdot\right) \stackrel{\mathrm{v}}{\rightarrow} v_{X, Y}
$$

as $t$ tends to $\infty$, for a nonnull Radon measure $v_{\boldsymbol{X}, \boldsymbol{Y}}$ on $\mathbb{E}_{2 d}$. Define the map $\Pi: \mathbb{R}^{d} \times \mathbb{R}^{d} \rightarrow \mathbb{R}^{d}$ by $\Pi(\boldsymbol{x}, \boldsymbol{y})=\boldsymbol{y} \cdot \boldsymbol{x}$. We assume that the measure $v_{\boldsymbol{X}, \boldsymbol{Y}} \circ \Pi^{-1}$ is not identically 0 . This means 
that there exists one index $i$ such that the pair $\left(X_{i}, Y_{i}\right)$ is asymptotically dependent, i.e.

$$
\text { there exists } i \in\{1, \ldots, d\} \text { such that } v_{X, Y}^{(i)}\left((0, \infty]^{2}\right)>0 \text {, }
$$

where $v_{\boldsymbol{X}, \boldsymbol{Y}}^{(i)}$ is defined as the restriction of $v$ to the $i$ th coordinates in $\boldsymbol{x}$ and $\boldsymbol{y}$. For instance, when $i=1, v^{(1)}(A)=v_{2 d}\left(A \times[-\infty, \infty]^{2 d-2}\right)$ for any $A \in \mathscr{B}_{2}$. The normalizing functions $a$ and $b$ are regularly varying with indices respectively denoted by $1 / \alpha$ and $1 / \beta$ for some positive real numbers $\alpha$ and $\beta$.

Theorem 4. Suppose that assumptions (12) and (13) hold. Then the random vector $\boldsymbol{Y} \cdot \boldsymbol{X} \in$ $\operatorname{MRV}\left(\alpha \beta /(\alpha+\beta), a b, v_{X, Y} \circ \Pi^{-1}\right)$, i.e.

$$
t \mathrm{P}\left(\boldsymbol{Y} \cdot \boldsymbol{X} \in a(t) b(t) \cdot \stackrel{\mathrm{v}}{\rightarrow} v_{\boldsymbol{X}, \boldsymbol{Y}} \circ \Pi^{-1}\right.
$$

as t tends to $\infty$.

In the particular case $Y_{1}=\cdots=Y_{d}$, this result can be found in Resnick (2007, Proposition 7.6).

Remark 5. Traditional models include an assumption of regular variation on the $\exp \left(Y_{i}\right) \mathrm{s}$ instead of the $Y_{i} \mathrm{~s}$, for instance, when the $Y_{i} \mathrm{~s}$ are log-normal. As a consequence, assuming that the discount factors have heavy tails might be considered as a strong hypothesis in a usual context. However, the model defined by (12) and (13) yields quite light tails for large values of $\beta$ and allows dependence between the $\boldsymbol{X}$ and $\boldsymbol{Y}$ components.

\section{Application to risk measures}

The link between a Breiman-type result and asymptotics for the ruin probability is highlighted in Subsection 4.1. The equivalents that we obtain depend on the ratio $Q(\boldsymbol{Y} \cdot \boldsymbol{X})$, which is not always explicitly known. Since numerical approximations can be done, we show some patterns in Subsection 4.2 in the case of the asymmetric logistic measure. Finally, in Subsection 4.3, we apply our results to the value at risk.

\subsection{Ruin probability}

Recall that the ruin probability associated to model (1) is defined by

$$
\psi(x, d)=\mathrm{P}\left(\max _{1 \leq k \leq d} \sum_{i=1}^{k} X_{i} Y_{i}>x\right)
$$

and that, for any random vector $\boldsymbol{Z}$, we have defined

$$
Q(\boldsymbol{Z})=\lim _{x \rightarrow \infty} \frac{\mathrm{P}\left(\sum_{i=1}^{d} Z_{i}>x\right)}{\sum_{i=1}^{d} \mathrm{P}\left(Z_{i}>x\right)}
$$

when the limit exists.

The next result establishes that the first equivalence of (2) holds if the random vector $\left(X_{1} Y_{1}, \ldots, X_{d} Y_{d}\right)$ is MRV and if all the random variables $X_{i}$ have lower tails lighter than their upper tails. 
Theorem 5. Under the assumptions of either Theorem 1, 2, 3, or 4, if the components of $\boldsymbol{Y}$ are nonnegative and the components of $\boldsymbol{X}$ satisfy (6), then we have, as $x$ tends to $\infty$,

$$
\psi(x, d) \sim \mathrm{P}\left(\sum_{i=1}^{d} X_{i} Y_{i}>x\right) .
$$

The second equivalence in (2) is specific to the asymptotic independence context considered in Zhang et al. (2009) and does not hold in general. However, as soon as a Breiman-type result is valid, we can derive other asymptotics for (14) depending explicitly on the margins of $\boldsymbol{X}$ and on $Q(\boldsymbol{Y} \cdot \boldsymbol{X})$. We make these relations explicit in the following corollaries, whose proofs are deferred to Section 6. Let us introduce the two sets $T=\left\{z \in \overline{\mathbb{R}}^{d} \mid \sum_{i=1}^{d} z_{i}>1\right\}$ and $T_{i}=\left\{z \in \overline{\mathbb{R}}^{d} \mid z_{i}>1\right\}$.

Corollary 1. Under the assumptions of Theorem 1, if the components of $\boldsymbol{Y}$ are nonnegative and the components of $\boldsymbol{X}$ satisfy (6), then, as $x$ tends to $\infty$,

$$
\psi(x, d) \sim Q(\boldsymbol{Y} \cdot \boldsymbol{X}) \sum_{i=1}^{d} \mathrm{E}\left[Y_{i}^{\alpha}\right] \mathrm{P}\left(X_{i}>x\right),
$$

where

$$
Q(\boldsymbol{Y} \cdot \boldsymbol{X})=\frac{v_{Y}(T)}{\sum_{i=1}^{d} v_{Y}\left(T_{i}\right)}=\frac{\mathrm{E}\left[v\left(\boldsymbol{Y}^{-1} \cdot T\right)\right]}{\sum_{i=1}^{d} \mathrm{E}\left[Y_{i}^{\alpha}\right] v\left(T_{i}\right)} .
$$

Note that, for any deterministic vector $\boldsymbol{Y}$, the numerator $v_{Y}(T)$ of the middle term in (16) corresponds to the extreme risk index of the portfolio denoted by $\gamma_{\boldsymbol{Y}}$. See Mainik and Rüschendorf (2009) and the references therein for complements on this risk measure.

Corollary 2. Under the assumptions of Theorem 2, if the components of $\boldsymbol{Y}$ are nonnegative and the components of $\boldsymbol{X}$ satisfy (6), then, as $x$ tends to $\infty$,

$$
\psi(x, d) \sim Q(\boldsymbol{Y} \cdot \boldsymbol{X}) \sum_{i=1}^{d} \mathrm{P}\left(X_{i}>x\right) \int_{0}^{\infty} y_{i}^{\alpha} \mathrm{d} L(\boldsymbol{y}),
$$

where

$$
Q(\boldsymbol{Y} \cdot \boldsymbol{X})=\frac{v_{L}(T)}{\sum_{i=1}^{d} v_{L}\left(T_{i}\right)}=\frac{\int v\left(\boldsymbol{y}^{-1} \cdot T\right) \mathrm{d} L(\boldsymbol{y})}{\sum_{i=1}^{d} v\left(T_{i}\right) \int y_{i}^{\alpha} \mathrm{d} L(\boldsymbol{y})}
$$

Remark 6. Corollary 1 recovers the results (for the finite time horizon) of Zhang et al. (2009). Indeed, under Corollary 1, as well as under Corollary 2, it can easily be proven that the components of $\boldsymbol{Y} \cdot \boldsymbol{X}$ are asymptotically independent as soon as those of $\boldsymbol{X}$ are asymptotically independent, so that $Q(\boldsymbol{Y} \cdot \boldsymbol{X})=1$. This comes from linearity properties combined with the fact that, for any vector $\boldsymbol{y}$ with positive components, any $i \in\{1, \ldots, d\}$, and any subset $A$ of $\mathbb{E}_{d}$, we have

$$
\left(y^{-1} \cdot A\right) \cap \mathbb{R} e_{i}=y^{-1} \cdot\left(A \cap \mathbb{R} e_{i}\right),
$$

where $\mathbb{R} e_{i}=\left\{r e_{i}, r \in \mathbb{R}\right\}$ and $e_{i}$ is the $i$ th canonical basis vector of $\mathbb{R}^{d}$. 
Corollary 3. Under the assumptions of Theorem 3, if the components of $\boldsymbol{Y}$ are nonnegative and the components of $\boldsymbol{X}$ satisfy (6), then, as $x$ tends to $\infty$,

$$
\psi(x, d) \sim \mathrm{P}\left(X_{1}>x\right) \sum_{i=1}^{d} \mathrm{E}\left[Y_{i}^{\alpha}\right] .
$$

In Corollary 3, the independence of the claim amounts $X_{i}$ yields $Q(\boldsymbol{Y} \cdot \boldsymbol{X})=1$, so that actuarial and financial information are separated in (18).

Corollary 4. Under the assumptions of Theorem 4, we have, as $x$ tends to $\infty$,

$$
\psi(x, d) \sim Q(\boldsymbol{Y} \cdot \boldsymbol{X}) \sum_{i=1}^{d} \mathrm{P}\left(X_{i} Y_{i}>x\right)
$$

where

$$
Q(\boldsymbol{Y} \cdot \boldsymbol{X})=\frac{v_{X, Y} \circ \Pi^{-1}(T)}{\sum_{i=1}^{d} v_{X, Y} \circ \Pi^{-1}\left(T_{i}\right)}
$$

Let us make some comments on the constant $Q(\boldsymbol{Y} \cdot \boldsymbol{X})$. We know from Lemma 2 that, under the assumptions of any of the previous corollaries, we have

$$
d^{-(1-\delta)_{+}} \leq Q(\boldsymbol{Y} \cdot \boldsymbol{X}) \leq d^{(\delta-1)_{+}},
$$

with $\delta=\alpha$ under Corollaries 1 and 2 and $\delta=\alpha \beta /(\alpha+\beta)$ under Corollary 4. Consequently, if $\delta \in(0,1]$ then $Q(\boldsymbol{Y} \cdot \boldsymbol{X})$ is between $d^{-(1-\delta)}$ and 1 , so the range between the two bounds is less than 1 , no matter the dimension $d$. Roughly speaking, the sum of probabilities is asymptotically smaller than the probability of the sum. However, it is possible to derive bounds depending on the moments of $\boldsymbol{Y}$. Let $Y_{(1)}$ and $Y_{(d)}$ be the smallest and the greatest component of $\boldsymbol{Y}$, respectively. For instance, Corollary 1 yields

$$
\frac{\sum_{i=1}^{d} v\left(T_{i}\right)}{\left(1 / \mathrm{E}\left[Y_{(1)}^{\alpha}\right]\right) \sum_{i=1}^{d} \mathrm{E}\left[Y_{i}^{\alpha}\right] v\left(T_{i}\right)} Q(\boldsymbol{X}) \leq Q(\boldsymbol{Y} \cdot \boldsymbol{X}) \leq \frac{\sum_{i=1}^{d} v\left(T_{i}\right)}{\left(1 / \mathrm{E}\left[Y_{(d)}^{\alpha}\right]\right) \sum_{i=1}^{d} \mathrm{E}\left[Y_{i}^{\alpha}\right] v\left(T_{i}\right)} Q(\boldsymbol{X})
$$

These bounds are particularly interesting because they allow us to separate the financial information contained in $\boldsymbol{Y}$ and the actuarial part given by $\boldsymbol{X}$ and $\nu$. The previous upper and lower bounds can also be relaxed and lead to

$$
\frac{\mathrm{E}\left[Y_{(1)}^{\alpha}\right]}{\max _{i=1, \ldots, d} \mathrm{E}\left[Y_{i}^{\alpha}\right]} Q(\boldsymbol{X}) \leq Q(\boldsymbol{Y} \cdot \boldsymbol{X}) \leq \frac{\mathrm{E}\left[Y_{(d)}^{\alpha}\right]}{\min _{i=1, \ldots, d} \mathrm{E}\left[Y_{i}^{\alpha}\right]} Q(\boldsymbol{X}) .
$$

Analogous bounds may be obtained under the assumptions of Corollary 2:

$$
\frac{\int_{[0, \infty]^{d}} y_{(1)}^{\alpha} \mathrm{d} L(\boldsymbol{y})}{\max _{i=1, \ldots, d} \int_{[0, \infty]^{d}} y_{i}^{\alpha} \mathrm{d} L(\boldsymbol{y})} Q(\boldsymbol{X}) \leq Q(\boldsymbol{Y} \cdot \boldsymbol{X}) \leq \frac{\int_{[0, \infty]^{d}} y_{(d)}^{\alpha} \mathrm{d} L(\boldsymbol{y})}{\min _{i=1, \ldots, d} \int_{[0, \infty]^{d}} y_{i}^{\alpha} \mathrm{d} L(\boldsymbol{y})} Q(\boldsymbol{X}) .
$$

When the limit measure $v$ of $\boldsymbol{X}$ is given, numerical bounds for $Q(\boldsymbol{Y} \cdot \boldsymbol{X})$ can thus be obtained from the preceding inequalities. This relies on the computation of the value of $Q(X)$, which is illustrated in the following section for the bivariate logistic dependence case. 


\subsection{Illustrative features of $Q(X)$}

In this subsection we illustrate the behavior of the term $Q(X)$ in the bivariate asymmetric logistic case. For other examples, we refer the reader to, e.g. Alink et al. (2004), Barbe et al. (2006), Kortschak and Albrecher (2009), and Embrechts et al. (2009a), (2009b). Recall that, when $d=2, Q(\boldsymbol{X})$ may be written in terms of the limit measure $v$ as follows:

$$
Q(\boldsymbol{X})=\frac{v(T)}{v\left(T_{1}\right)+v\left(T_{2}\right)} .
$$

Here $T=\left\{z \in \overline{\mathbb{R}}^{2} \mid z_{1}+z_{2}>1\right\}$ and $T_{i}=\left\{z \in \overline{\mathbb{R}}^{2} \mid z_{i}>1\right\}$.

We consider a random vector $\boldsymbol{X}=\left(X_{1}, X_{2}\right)$ with the following bivariate extreme value distribution:

- both margins are identically distributed from the Fréchet $(\mu=0, \sigma=1, \xi=\alpha)$ distribution, which means that there exists some positive $\alpha$ such that, for any positive $x$, we have $F_{X_{i}}(x)=\mathrm{P}\left(X_{i} \leq x\right)=\exp \left\{-x^{-\alpha}\right\}$;

- the dependence between the margins of $\boldsymbol{X}$ is characterized by a function $\ell$ satisfying the conditions of a stable tail dependence function (see, e.g. Conditions L1, L2, and L3 of Beirlant et al. (2004, p. 257)), so that the distribution function of $\boldsymbol{X}$ can be written as

$$
F_{\boldsymbol{X}}(\boldsymbol{x})=\mathrm{P}(\boldsymbol{X} \leq \boldsymbol{x})=\exp \left\{-\ell\left(x_{1}^{-\alpha}, x_{2}^{-\alpha}\right)\right\}
$$

We fix the stable tail dependence function to be the asymmetric logistic defined by

$$
\ell_{\psi_{1}, \psi_{2}, r}\left(x_{1}, x_{2}\right)=\left(1-\psi_{1}\right) x_{1}+\left(1-\psi_{2}\right) x_{2}+\left\{\left(\psi_{1} x_{1}\right)^{1 / r}+\left(\psi_{2} x_{2}\right)^{1 / r}\right\}^{r}
$$

for any $0<r \leq 1$ and $0 \leq \psi_{1}, \psi_{2} \leq 1$. This parametric model has been widely used, and is, e.g. presented in Section 9.2.2 of Beirlant et al. (2004).

We compute $Q(\boldsymbol{X})$ by standard upper and lower Riemann approximations. In dimension two, this procedure is very accurate: lower and upper curves are so close that they are indistinguishable; see Figures 1 and 2.

In the special case where $\psi_{1}=\psi_{2}=1$, the distribution of $\boldsymbol{X}$ is the bivariate symmetric logistic model with Fréchet margins. The strength of dependence between the components of $\boldsymbol{X}$ is a decreasing function of $r$. In particular, the independence and the total positive dependence respectively correspond to $r=1$ and $r \rightarrow 0$. The values of $Q(\boldsymbol{X})$ for this model are given in Figure 1 as functions of $r$ and $\alpha$.

Several aspects of these plots have already been observed in the literature for other models: $Q(\boldsymbol{X})$ tends to 1 as $r$ tends to $1 ; Q(\boldsymbol{X})$ tends to $2^{\alpha-1}$ as $r$ tends to 0 ; for $\alpha<1, Q(\boldsymbol{X})$ is strictly increasing in $r$ and, for $\alpha>1, Q(\boldsymbol{X})$ is strictly decreasing in $r$; for all $r, Q(\boldsymbol{X})$ is strictly increasing in $\alpha$.

More generally, the patterns of $Q(\boldsymbol{X})$ when $\psi_{1}$ and $\psi_{2}$ are in $(0,1)$ are presented in Figure 2. For specific values of $\alpha, \psi_{1}$, and $r$, we plot $Q(\boldsymbol{X})$ as a function of the parameter $\psi_{2}$.

Again, we observe several elements of these plots: $Q(\boldsymbol{X})$ is strictly decreasing or increasing in $\psi_{1}$ or $\psi_{2}$ when $\alpha<1$ or, respectively, $\alpha>1$; for any values of $\psi_{1}$ and $\psi_{2}, Q(\boldsymbol{X})$ is strictly increasing or decreasing in $r$ when $\alpha<1$ or, respectively, $\alpha>1$.

The computation of numerical approximations of $Q(\boldsymbol{X})$ can also be performed for values of $d$ larger than two. Note however that this procedure becomes quickly time consuming when $d$ is large. 

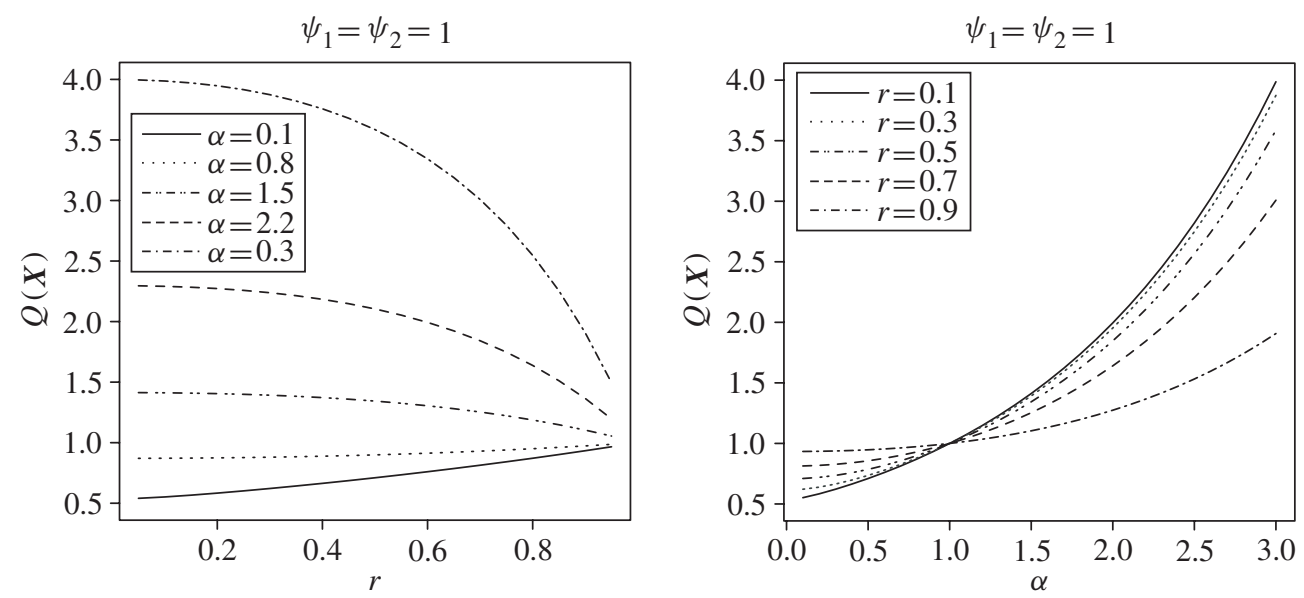

FIGURE 1: Values of $Q(\boldsymbol{X})$ as a function of $r$ or $\alpha$ when $\boldsymbol{X}$ has the symmetric logistic dependence structure $\ell_{1,1, r}$ and Fréchet $(\alpha)$ margins. Left: $Q(\boldsymbol{X})$ as a function of the dependence parameter $r \in(0,1)$ for values of $\alpha$ among $\{0.1,0.8,1.5,2.2,3\}$. Right: $Q(\boldsymbol{X})$ as a function of $\alpha \in(0.1,3)$ for values of $r$ among $\{0.1,0.3,0.5,0.7,0.9\}$.
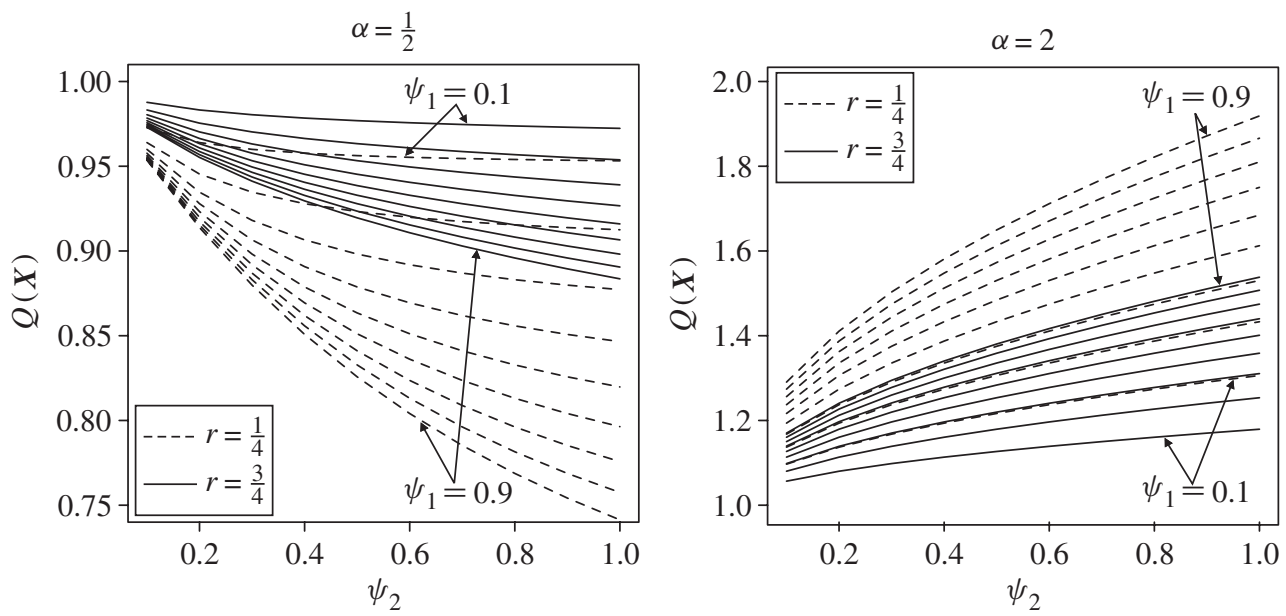

FiguRe 2: Values of $Q(\boldsymbol{X})$ as a function of $\psi_{2}$ when $\boldsymbol{X}$ has the asymmetric dependence structure $\ell_{\psi_{1}, \psi_{2}, r}$ and Fréchet $(\alpha)$ margins. Left: $\psi_{1}=0.1, \ldots, 0.9$ (in order from top to bottom) for $\alpha=\frac{1}{2}$ and $r=\frac{1}{4}, \frac{3}{4}$. Right: $\psi_{1}=0.1, \ldots, 0.9$ (in order from bottom to top) for $\alpha=2$ and $r=\frac{1}{4}, \frac{3}{4}$.

\subsection{Value at risk}

Another classical risk measure is the value at risk, defined as follows. Given $p \in(0,1)$ and a random variable $Z$, the value at risk of $Z$ at level $p$, denoted by $\operatorname{VaR}_{p}(Z)$, is the $p$ th quantile of $Z$, i.e. $\operatorname{VaR}_{p}(Z)=\inf \{z \mid \mathrm{P}(Z \leq z) \geq p\}$. An important feature of risk measures is the property of subadditivity or superadditivity, which can be written for the value at risk in terms of the ratio

$$
R_{Z}^{(p)}=\frac{\operatorname{VaR}_{p}\left(\sum_{i=1}^{d} Z_{i}\right)}{\sum_{i=1}^{d} \operatorname{VaR}_{p}\left(Z_{i}\right)},
$$


where $\boldsymbol{Z}$ denotes the vector $\left(Z_{1}, \ldots, Z_{d}\right)^{\top}$. We say that subadditivity or superadditivity holds for $\operatorname{VaR}_{p}(Z)$ if $R_{Z}^{(p)} \leq 1$ or $R_{Z}^{(p)}>1$, respectively. Analogously, asymptotic subadditivity or asymptotic superadditivity holds for $\operatorname{VaR}_{p}(Z)$ if

$$
\lim _{p \rightarrow 0} R_{Z}^{(p)} \leq 1 \quad \text { or } \quad \lim _{p \rightarrow 0} R_{Z}^{(p)}>1,
$$

respectively.

In the following corollary we summarize the consequences of the asymptotics obtained in the previous subsection on the value at risk, which in particular allows us to discuss explicit cases where asymptotic subadditivity and asymptotic superadditivity of $\operatorname{VaR}_{p}(\boldsymbol{Y} \cdot \boldsymbol{X})$ holds. Analogous results are given in Embrechts et al. (2009a) in the case of elliptical claim amounts.

Corollary 5. Assume that the components of $\boldsymbol{Y}$ are nonnegative and that the components of $\boldsymbol{X}$ satisfy (6). Then, under the assumptions of Theorem 1,

$$
\lim _{p \rightarrow 1} R_{\boldsymbol{Y} \cdot \boldsymbol{X}}^{(p)}=Q(\boldsymbol{Y} \cdot \boldsymbol{X})^{1 / \alpha} \frac{\left(\sum_{i=1}^{d} \mathrm{E}\left[Y_{i}^{\alpha}\right] v\left(T_{i}\right)\right)^{1 / \alpha}}{\sum_{i=1}^{d}\left(\mathrm{E}\left[Y_{i}^{\alpha}\right] v\left(T_{i}\right)\right)^{1 / \alpha}} .
$$

Under the assumptions of Theorem 2,

$$
\lim _{p \rightarrow 1} R_{\boldsymbol{Y} \cdot \boldsymbol{X}}^{(p)}=Q(\boldsymbol{Y} \cdot \boldsymbol{X})^{1 / \alpha} \frac{\left(\sum_{i=1}^{d} \int_{0}^{\infty} y_{i}^{\alpha} L(\mathrm{~d} \boldsymbol{y}) v\left(T_{i}\right)\right)^{1 / \alpha}}{\sum_{i=1}^{d}\left(\int_{0}^{\infty} y_{i}^{\alpha} L(\mathrm{~d} \boldsymbol{y}) v\left(T_{i}\right)\right)^{1 / \alpha}} .
$$

Under the assumptions of Theorem 3,

$$
\lim _{p \rightarrow 1} R_{\boldsymbol{Y} \cdot \boldsymbol{X}}^{(p)}=\frac{\left(\sum_{i=1}^{d} \mathrm{E}\left[Y_{i}^{\alpha}\right] v\left(T_{i}\right)\right)^{1 / \alpha}}{\sum_{i=1}^{d}\left(\mathrm{E}\left[Y_{i}^{\alpha}\right] v\left(T_{i}\right)\right)^{1 / \alpha}} .
$$

Under the assumptions of Theorem 4,

$$
\lim _{p \rightarrow 1} R_{\boldsymbol{Y} \cdot \boldsymbol{X}}^{(p)}=Q(\boldsymbol{Y} \cdot \boldsymbol{X})^{1 / \alpha} \frac{\left(\sum_{i=1}^{d} v_{\boldsymbol{X}, \boldsymbol{Y}} \circ \Pi^{-1}\left(T_{i}\right)\right)^{1 / \alpha}}{\sum_{i=1}^{d}\left(v_{\boldsymbol{X}, \boldsymbol{Y}} \circ \Pi^{-1}\left(T_{i}\right)\right)^{1 / \alpha}} .
$$

The proof of Corollary 5 is postponed until Section 6 .

Remark 7. Corollary 5 allows us to deduce in some cases asymptotic subadditivity or superadditivity. For instance, under the assumptions of Theorem 1, and using (16), we obtain

$$
\lim _{p \rightarrow 1} R_{\boldsymbol{Y} \cdot \boldsymbol{X}}^{(p)}=\frac{\left\{\mathrm{E}\left[v\left(\boldsymbol{Y}^{-1} \cdot T\right)\right]\right\}^{1 / \alpha}}{\sum_{i=1}^{d}\left(\mathrm{E}\left[Y_{i}^{\alpha}\right] v\left(T_{i}\right)\right)^{1 / \alpha}} .
$$

If the $Y_{i}$ s are identically distributed and the measure $v$ is symmetric, we obtain

$$
\lim _{p \rightarrow 1} R_{\boldsymbol{Y} \cdot \boldsymbol{X}}^{(p)} \geq \frac{\left\{v_{\boldsymbol{Y}}\left([0, \mathbf{1}]^{c}\right)\right\}^{1 / \alpha}}{d\left\{\mathrm{E}\left[Y_{1}^{\alpha}\right] v\left(T_{1}\right)\right\}^{1 / \alpha}} \geq \frac{\{\ell(1, \ldots, 1)\}^{1 / \alpha}}{d} .
$$

Now, if we take the stable tail dependence function $\ell$ to be the symmetric logistic, that is, $\ell(\boldsymbol{u})=\left(\sum_{i=1}^{d} u_{i}^{1 / \beta}\right)^{\beta}$ with $0<\beta \leq 1$, we obtain $\lim _{p \rightarrow 1} R_{\boldsymbol{Y} \cdot \boldsymbol{X}}^{(p)} \geq d^{\beta / \alpha-1}$. Hence, asymptotic superadditivity follows as soon as $\beta \geq \alpha$.

An example of asymptotic subadditivity can be derived from Corollary 5, under the assumption of Theorem 3. When the $Y_{i} \mathrm{~s}$ are identically distributed and the measure $v$ is symmetric, we can easily prove that $\lim _{p \rightarrow 1} R_{\boldsymbol{Y} \cdot \boldsymbol{X}}^{(p)}=d^{1 / \alpha-1}$. Asymptotic subadditivity is thus equivalent in this framework to the condition $\alpha>1$. 


\section{Concluding comments and discussion}

In this paper, discrete-time risk models with finite time horizon have been considered, and asymptotics for risk measures, as ruin probability or value at risk, have been obtained under different dependence settings for the claim amounts and discount factors. The key role of the multivariate regularly varying (MRV) setting has been highlighted, as well as the usefulness of Breiman-type results. This allowed us to generalize (for the finite time horizon) the result of Zhang et al. (2009) outside the asymptotic independence of the claim amounts, and outside the independence of claim amounts and discount factors.

A specific parameter $Q(\boldsymbol{Y} \cdot \boldsymbol{X})$ arises from these asymptotics, for which explicit bounds have been provided in terms of the limit measure of $\boldsymbol{X}$ and the characteristics of the discount factors $\boldsymbol{Y}$. These bounds are easily numerically computable as long as the time horizon $d$ is not too large. Dealing with very high dimensions still represents a challenging numerical problem. Ideas coming from the algorithms developed in Arbenz et al. (2011) could be promising for this task.

Another important issue is to measure the accuracy of the approximations stated in Theorem 5 and its corollaries. Such a problem requires MRV hypotheses with second-order conditions, as formulated in the univariate case in Degen et al. (2010). This will be the subject of future work.

\section{Proofs}

Proof of Lemma 1. We prove the first assertion of Lemma 1 by induction. Let $K$ be a relatively compact set of $\mathbb{E}_{d-1}$ such that $\nu(\partial K)=0$, and let $\varepsilon>0$. By assumption, convergence (3) implies that

$$
\lim _{t \rightarrow \infty} t \mathrm{P}\left(a(t)^{-1} \boldsymbol{X} \in K \times(-\infty,-\varepsilon]\right)=v(K \times(-\infty,-\varepsilon]) .
$$

Furthermore,

$$
t \mathrm{P}\left(a(t)^{-1} \boldsymbol{X} \in K \times(-\infty,-\varepsilon]\right) \leq t \mathrm{P}\left(X_{d}<-a(t) \varepsilon\right) \rightarrow 0
$$

therefore, $v(K \times(-\infty,-\varepsilon])=0$, so the support of $v$ is included in $\mathbb{R}^{d-1} \times[0, \infty) \backslash\{\boldsymbol{0}\}$. The rest of the induction argument is along the same lines. It is then obvious that the measure $H_{\|\cdot\|}$ is concentrated on $\mathbb{S}_{\|\cdot\|}^{d-1} \cap[0, \infty]^{d}$. Now, for simplicity, we consider the following sets:

$$
\begin{aligned}
T_{\max } & =\left\{z \in \overline{\mathbb{R}}^{d} \mid \max _{1 \leq k \leq d} \sum_{i=1}^{k} z_{i}>1\right\}, \\
T & =\left\{z \in \overline{\mathbb{R}}^{d} \mid \sum_{i=1}^{d} z_{i}>1\right\}, \\
T_{+} & =\left\{z \in[0, \infty]^{d} \mid \sum_{i=1}^{d} z_{i}>1\right\} .
\end{aligned}
$$

The last assertion of Lemma 1 may then be written as $v\left(T_{\max }\right) / v(T)=v\left(T^{+}\right) / v(T)=1$, which is obvious when $v$ is concentrated on the nonnegative quadrant.

Proof of Lemma 2. Recall that $H_{1}$ denotes the spectral measure of $\boldsymbol{X}$ with respect to the $\ell_{1}$-norm. This is a probability measure concentrated on $\left\{w \in \mathbb{S}_{1}^{d-1} \mid w_{i} \geq 0,1 \leq i \leq d\right\}$. 
The choice of the $\ell_{1}$-norm yields $\lim _{t \rightarrow \infty} t \mathrm{P}\left(\sum_{i=1}^{d} X_{i}>a(t)\right)=1$. Furthermore, for any $\boldsymbol{y} \in[0, \infty]^{d}$, from (5) we obtain

$$
v\left(\left\{z \in[0, \infty]^{d} \backslash\{\mathbf{0}\} \mid \sum_{i=1}^{d} y_{i} z_{i}>1\right\}\right)=\int_{\mathbb{S}_{1}^{d-1}}\left(\sum_{i=1}^{d} y_{i} w_{i}\right)^{\alpha} H_{1}(\mathrm{~d} \boldsymbol{w}),
$$

which implies in particular that

$$
\lim _{t \rightarrow \infty} t \mathrm{P}\left(X_{i}>a(t)\right)=\int_{\mathbb{S}_{1}^{d-1}} w_{i}^{\alpha} H_{1}(\mathrm{~d} w)
$$

Consequently, we obtain

$$
\lim _{t \rightarrow \infty} t \sum_{i=1}^{d} \mathrm{P}\left(X_{i}>a(t)\right)=\sum_{i=1}^{d} \int_{\mathbb{S}_{1}^{d-1}} w_{i}^{\alpha} H_{1}(\mathrm{~d} \boldsymbol{w}),
$$

so that

$$
Q(\boldsymbol{X})=\frac{1}{\sum_{i=1}^{d} \int_{\mathbb{S}_{1}^{d-1}} w_{i}^{\alpha} H_{1}(\mathrm{~d} \boldsymbol{w})}
$$

If $\alpha>1$ then the function $x \rightarrow x^{\alpha}$ is convex. By Jensen's inequality we have

$$
\frac{1}{d} \sum_{i=1}^{d} \int_{\mathbb{S}_{1}^{d-1}} w_{i}^{\alpha} H_{1}(\mathrm{~d} \boldsymbol{w}) \geq\left\{\frac{1}{d} \sum_{i=1}^{d} \int_{\mathbb{S}_{1}^{d-1}} w_{i} H_{1}(\mathrm{~d} \boldsymbol{w})\right\}^{\alpha}=\left\{\frac{1}{d} \int_{\mathbb{S}_{1}^{d-1}} \sum_{i=1}^{d} w_{i} H_{1}(\mathrm{~d} \boldsymbol{w})\right\}^{\alpha}=d^{-\alpha}
$$

If $\alpha<1$ then, again by Jensen's inequality, the reverse bound holds:

$$
d^{-\alpha}=\left\{\frac{1}{d} \sum_{i=1}^{d} \int_{\mathbb{S}_{1}^{d-1}} w_{i} H_{1}(\mathrm{~d} \boldsymbol{w})\right\}^{\alpha} \geq \frac{1}{d} \sum_{i=1}^{d} \int_{\mathbb{S}_{1}^{d-1}} w_{i}^{\alpha} H_{1}(\mathrm{~d} \boldsymbol{w}) .
$$

On the other hand, if $\alpha>1$ then $w^{\alpha} \leq w$ for $w \in[0,1]$ so

$$
\sum_{i=1}^{d} \int_{\mathbb{S}_{1}^{d-1}} w_{i}^{\alpha} H_{1}(\mathrm{~d} \boldsymbol{w}) \leq \sum_{i=1}^{d} \int_{\mathbb{S}_{1}^{d-1}} w_{i} H_{1}(\mathrm{~d} \boldsymbol{w})=1 .
$$

The reverse inequality obviously holds for $\alpha<1$. Gathering these bounds yields (7). The equality $Q(\boldsymbol{X})=1$ when the components of $\boldsymbol{X}$ are asymptotically independent follows from the fact that $H_{1}$ is then concentrated on the axes.

\subsection{Proof of Theorem 2}

In order to simplify the notation, we denote by $\|\cdot\|$ a given norm on a Euclidean space, and, for any $q \times d$ matrix $M$, the induced matrix norm is $\|M\|=\sup _{\|x\|=1}\|M x\|$. For a $q \times d$ matrix $M$ and a set $K \subset \mathbb{R}^{q}$, we define

$$
M^{-1} \cdot K=\left\{x \in \mathbb{R}^{d} \mid M x \in K\right\} .
$$


In order to prove Theorem 2, we state and prove a more general result. We use the concept of asymptotic independence introduced in Maulik et al. (2002). Assume that $\boldsymbol{M}$ is a random matrix of size $q \times d$ and $\boldsymbol{X} \in \mathbb{R}^{d}$ is a random vector satisfying

$$
t \mathrm{P}\left(\left(\frac{\boldsymbol{X}}{a(t)}, \boldsymbol{M}\right) \in \cdot\right) \stackrel{\mathrm{v}}{\rightarrow}(v \times G)(\cdot)
$$

as $t$ tends to $\infty$ on $\mathbb{E}_{d} \times[-\infty, \infty]^{q d}$, where $v$ is a Radon measure on $\mathbb{E}_{d}$ not concentrated at $\infty$ and $G$ is a probability measure on $[-\infty, \infty]^{q d}$. This implies that $v$ is homogeneous with positive index $\alpha$, say, and we can still choose the normalizing function $a$ such that

$$
\lim _{t \rightarrow \infty} t \mathrm{P}(\|X\|>a(t))=1 .
$$

Assume also that there exists $\delta>0$ such that

$$
\begin{gathered}
\lim _{\varepsilon \rightarrow 0} \limsup _{t \rightarrow \infty} t \mathrm{E}\left[\left(\frac{\|\boldsymbol{M}\|\|\boldsymbol{X}\|}{a(t)}\right)^{\delta} \mathbf{1}_{\{\|\boldsymbol{X}\| \leq \varepsilon a(t)\}}\right]=0, \\
\int_{[-\infty, \infty]^{q d}}\|M\|^{\alpha} G(\mathrm{~d} M)<\infty .
\end{gathered}
$$

Let $v_{G}$ denote the measure defined on $\mathbb{E}_{q}$ by

$$
\begin{aligned}
v_{G}(K) & =v \otimes G(\{(\boldsymbol{x}, M) \mid M \boldsymbol{x} \in K\}) \\
& =\int_{[-\infty, \infty]^{q d}} v\left(M^{-1} \cdot K\right) G(\mathrm{~d} M) \\
& =\mathrm{E}\left[v\left(\boldsymbol{M}^{*-1} \cdot K\right)\right],
\end{aligned}
$$

where $\boldsymbol{M}^{*}$ is a random matrix with distribution $G$.

Theorem 6. Assume that assumptions (20), (21), and (22) hold. Then

$$
t \mathrm{P}\left(a(t)^{-1} \boldsymbol{M} \boldsymbol{X} \in \cdot\right) \stackrel{\mathrm{v}}{\rightarrow} v_{G}
$$

as $t$ tends to $\infty$.

Proof. Let $K$ be relatively compact in $\mathbb{E}_{q}$ such that $v_{G}(\partial K)=0$. Fix some real number $s>0$, and write

$$
\mathrm{P}(\boldsymbol{M} \boldsymbol{X} \in a(t) K)=\mathrm{P}(\boldsymbol{M} \boldsymbol{X} \in a(t) K,\|\boldsymbol{M}\| \leq s)+\mathrm{P}(\boldsymbol{M} \boldsymbol{X} \in a(t) K,\|\boldsymbol{M}\|>s) .
$$

Since $K$ is relatively compact in $\mathbb{E}_{q}$, there exists $\kappa>0$ such that $\|x\| \geq \kappa$ for all $\boldsymbol{x} \in K$. Thus, $\|M\| \leq s$ and $M \boldsymbol{x} \in K$ imply that $\|\boldsymbol{x}\| \geq s^{-1} \kappa$; thus, assumption (20) implies that

$$
\lim _{t \rightarrow \infty} t \mathrm{P}(\boldsymbol{M} \boldsymbol{X} \in a(t) K,\|\boldsymbol{M}\| \leq s)=\mathrm{E}\left[v\left(\boldsymbol{M}^{*-1} \cdot K\right) \mathbf{1}_{\{\|\boldsymbol{M}\| \leq s\}}\right] .
$$

By the homogeneity of $v$, condition (22), and the monotone convergence theorem, it holds that

$$
\lim _{s \rightarrow \infty} \mathrm{E}\left[v\left(\boldsymbol{M}^{*-1} \cdot K\right) \mathbf{1}_{\{\|\boldsymbol{M}\| \leq s\}}\right]=\mathrm{E}\left[v\left(\boldsymbol{M}^{*-1} \cdot K\right)\right] .
$$


Next, since $\boldsymbol{y} \in K$ implies that $\|\boldsymbol{y}\| \geq \kappa$, we have, for $s \geq 1$ and $\varepsilon>0$,

$$
\begin{aligned}
\mathrm{P}(\boldsymbol{M} \boldsymbol{X} \in a(t) K,\|\boldsymbol{M}\|>s) & \leq \mathrm{P}(\|\boldsymbol{M}\|\|\boldsymbol{X}\|>\kappa a(t),\|\boldsymbol{M}\|>s) \\
& \leq \mathrm{P}(\|\boldsymbol{X}\|>\varepsilon a(t),\|\boldsymbol{M}\|>s)+\mathrm{P}\left(\frac{\|\boldsymbol{M}\|\|\boldsymbol{X}\|}{a(t)}>\kappa, \frac{\|\boldsymbol{X}\|}{a(t)} \leq \varepsilon\right) .
\end{aligned}
$$

By assumption (20),

$$
\lim _{t \rightarrow \infty} t \mathrm{P}(\|\boldsymbol{X}\|>\varepsilon a(t),\|\boldsymbol{M}\|>s)=\varepsilon^{-\alpha} \mathrm{P}\left(\left\|\boldsymbol{M}^{*}\right\|>s\right),
$$

which can be made arbitrarily small by choosing $s$ large enough. By Markov's inequality and assumption (21),

$$
\begin{aligned}
\limsup _{t \rightarrow \infty} t \mathrm{P}\left(\frac{\|\boldsymbol{M}\|\|\boldsymbol{X}\|}{a(t)}>\kappa, \frac{\|\boldsymbol{X}\|}{a(t)} \leq \varepsilon\right) & \leq \kappa^{-\delta} \limsup _{t \rightarrow \infty} \mathrm{E}\left[\left(\frac{\|\boldsymbol{M}\|\|\boldsymbol{X}\|}{a(t)}\right)^{\delta} \mathbf{1}_{\{\|\boldsymbol{X}\| \leq \varepsilon a(t)\}}\right] \\
& =0 .
\end{aligned}
$$

Thus, we have proven that $\lim _{t \rightarrow \infty} t \mathrm{P}(\boldsymbol{M} \boldsymbol{X} \in a(t) K)=\mathrm{E}\left[v\left(\boldsymbol{M}^{*-1} \cdot K\right)\right]$ and this concludes the proof.

Proof of Theorem 3. It is a straightforward consequence of Hult and Samorodnitsky (2008, Theorem 3.1) for $p=1, A_{0}=0, A_{j}=Y_{j} \boldsymbol{e}_{j}$ and $Z_{j}=X_{j}$ for $j=1, \ldots, d$, and $A_{j}=0$ for $j>d$.

Proof of Theorem 4. Let $K$ be a relatively compact set in $\mathbb{E}_{d}$ such that $v_{X, Y} \circ \Pi^{-1}(\partial K)=0$. Then $v_{\boldsymbol{X}, \boldsymbol{Y}}\left(\partial \Pi^{-1} K\right)=0$, since $\Pi$ is continuous, which implies that $\partial \Pi^{-1}(K) \subset \Pi^{-1}(\partial K)$. Moreover, $\Pi^{-1}(K)$ is relatively compact in $\mathbb{E}_{2 d}$. To see this, we can choose some arbitrary norm and prove that, for some $\xi>0$, the set $K=\left\{x \in \mathbb{R}^{d} \mid\|x\|>\xi\right\}$ is such that $\Pi^{-1}(K)$ is bounded away from 0 in $\mathbb{E}_{2 d}$. Choose, for instance, the Euclidean norm in both $\mathbb{E}_{d}$ and $\mathbb{E}_{2 d}$. Then

$$
\|\boldsymbol{x} \cdot \boldsymbol{y}\|^{2}=\sum_{i=1}^{d} x_{i}^{2} y_{i}^{2} \leq \sum_{i=1}^{d}\left(x_{i}^{2}+y_{i}^{2}\right) y_{i}^{2} \leq \sum_{i=1}^{d}\left(x_{i}^{2}+y_{i}^{2}\right)^{2}=\|(\boldsymbol{x}, \boldsymbol{y})\|^{2} .
$$

Thus, if $(\boldsymbol{x}, \boldsymbol{y}) \in \Pi^{-1}(K)$ then $\|(\boldsymbol{x}, \boldsymbol{y})\| \geq\|\boldsymbol{x} \cdot \boldsymbol{y}\|>\xi$ and this proves that $\Pi^{-1}(K)$ is relatively compact in $\mathbb{E}_{2 d}$. Define $c(t)=a(t) b(t)$. Then,

$$
\begin{aligned}
\lim _{t \rightarrow \infty} t \mathrm{P}\left(\frac{\boldsymbol{Y} \cdot \boldsymbol{X}}{c(t)} \in K\right) & =\lim _{t \rightarrow \infty} t \mathrm{P}\left(\left(\frac{X_{i}}{a(t)}, \frac{Y_{i}}{b(t)}\right)_{i=1, \ldots, d} \in \Pi^{-1}(K)\right) \\
& =v_{\boldsymbol{X}, \boldsymbol{Y}} \circ \Pi^{-1}(K) .
\end{aligned}
$$

Proof of Theorem 5. We want to state (14). Under the assumptions of any of the theorems of Section 3, $\boldsymbol{Y} \cdot \boldsymbol{X}$ is MRV. Let $\mu$ denote the Radon measure associated with $\boldsymbol{Y} \cdot \boldsymbol{X}$, and let $a$ be the normalizing function. Then (14) is equivalent to

$$
\mu(T)=\mu\left(T_{\max }\right)=\mu\left(T_{+}\right),
$$

where $T, T_{\max }$, and $T_{+}$are as defined above. This holds as soon as the support of $\mu$ is included in $[0, \infty]^{d} \backslash\{\boldsymbol{0}\}$, which we now prove. By Lemma 1 , it suffices to prove that (6) holds for the 
vector $\boldsymbol{Y} \cdot \boldsymbol{X}$, i.e. for each $i=1, \ldots, d$,

$$
\lim _{x \rightarrow \infty} \frac{\mathrm{P}\left(X_{i} Y_{i}<-x\right)}{\mathrm{P}\left(X_{i} Y_{i}>x\right)}=0 .
$$

The latter equivalence holds under the assumptions of Theorems 1, 2, and 3 since $X_{i}$ and $Y_{i}$ are asymptotically independent, so that

$$
\mathrm{P}\left(X_{i} Y_{i}>x\right) \sim c_{i} \mathrm{P}\left(X_{i}>x\right) \text { and } \mathrm{P}\left(X_{i} Y_{i}<-x\right) \sim c_{i} \mathrm{P}\left(X_{i}<-x\right)
$$

in each of these cases, where $c_{i}=\mathrm{E}\left[Y_{i}^{\alpha}\right]$ under the assumptions of Theorems 1 and 3, and $c_{i}=\int_{0}^{\infty} y_{i}^{\alpha} L(\mathrm{~d} \boldsymbol{y})$ under the assumptions of Theorem 2 .

Under the assumptions of Theorem 4, we must prove that if $X$ and $Y$ are two jointly regularly varying random variables such that $Y$ is nonnegative and $X$ satisfies (6), then $X Y$ also satisfies (6). This clearly holds since, for any $x>0$,

$$
0=\lim _{t \rightarrow \infty} t \mathrm{P}(X<-a(t) x)=v_{X, Y}((-\infty,-x) \times[0, \infty)) .
$$

Thus, the support of $v_{X, Y}$ is included in $[0, \infty]^{2}$. This proves (23) and concludes the proof of (14) under the assumptions of Theorem 4, completing the proof of Theorem 5.

Proofs of Corollaries 1-4. We need to respectively prove (15), (17), (18), and (19). Note that (19) follows directly from the definition of $Q(\boldsymbol{X} \cdot \boldsymbol{Y})$ and is valid in all contexts. Thus, we only need to give further equivalents of $\sum_{i=1}^{d} \mathrm{P}\left(X_{i} Y_{i}>x\right)$.

- Under the assumptions of Theorems 1 and 3, $X_{i}$ and $Y_{i}$ are independent, and Breiman's theorem applies: $\mathrm{P}\left(X_{i} Y_{i}>x\right) \sim \mathrm{E}\left[Y_{i}^{\alpha}\right] \mathrm{P}\left(X_{i}>x\right)$. Under the assumptions of Theorem 3, the components of $\boldsymbol{Y} \cdot \boldsymbol{X}$ are asymptotically independent, as shown in (11), so a consequence of Lemma 2 is that $Q(\boldsymbol{Y} \cdot \boldsymbol{X})=1$.

- Under the assumptions of Theorem 2, it follows from conditions (8), (9), and (10) that $\mathrm{P}\left(X_{i} Y_{i}>x\right) \sim \int_{0}^{\infty} y_{i}^{\alpha} L(\mathrm{~d} \boldsymbol{y}) \mathrm{P}\left(X_{i}>x\right)$.

Proof of Corollary 5. The following lemma is useful to prove Corollary 5, and is a straightforward consequence of usual properties of inverses of regularly varying functions; see, e.g. Resnick (1987, Proposition 0.8(vi)). We state it here (without proof) for completeness.

Lemma 3. Let $0<\alpha<\infty$. If $X$ and $Y$ are two random variables with regularly varying upper tails with index $-\alpha$, then, for $0 \leq a \leq \infty$,

$$
\lim _{x \rightarrow \infty} \frac{\mathrm{P}(X>x)}{\mathrm{P}(Y>x)}=a \Longleftrightarrow \lim _{p \rightarrow 1} \frac{\operatorname{VaR}_{p}(X)}{\operatorname{VaR}_{p}(Y)}=a^{1 / \alpha} .
$$

Under the assumptions of either Theorem 1,2,3, or 4, we want to obtain the following common expression:

$$
\lim _{p \rightarrow 1} \frac{\operatorname{VaR}_{p}\left(\sum_{i=1}^{d} X_{i} Y_{i}\right)}{\sum_{i=1}^{d} \operatorname{VaR}_{p}\left(X_{i} Y_{i}\right)}=\{Q(\boldsymbol{Y} \cdot \boldsymbol{X}) D\}^{1 / \alpha}
$$

Here

$$
D=\frac{\sum_{i=1}^{d} \mu\left(T_{i}\right)}{\left\{\sum_{i=1}^{d} \mu\left(T_{i}\right)^{1 / \alpha}\right\}^{\alpha}}
$$


where $T_{i}=\left\{z \in \overline{\mathbb{R}}^{d} \mid z_{i}>1\right\}$ and $\mu$ denotes the limit measure of $\boldsymbol{Y} \cdot \boldsymbol{X}$. Define the random variable $Z$ via the relationship

$$
\operatorname{VaR}_{p} Z=\sum_{i=1}^{d} \operatorname{VaR}_{p}\left(X_{i} Y_{i}\right)
$$

Lemma 3 says that (24) is equivalent to

$$
\lim _{x \rightarrow \infty} \frac{\mathrm{P}\left(\sum_{i=1}^{d} X_{i} Y_{i}>x\right)}{\mathrm{P}(Z>x)}=Q(\boldsymbol{Y} \cdot \boldsymbol{X}) D,
$$

which is also equivalent, by the definition of $Q(\boldsymbol{Y} \cdot \boldsymbol{X})$, to

$$
\lim _{x \rightarrow \infty} \frac{\sum_{i=1}^{d} \mathrm{P}\left(X_{i} Y_{i}>x\right)}{\mathrm{P}(Z>x)}=D
$$

This limit $D$ can be made explicit using the change of variable $x=\gamma(t)$, where $\gamma(t)=$ $a(t)$ under the assumptions of Theorems $1-3$, and $\gamma(t)=a(t) b(t)$ under the assumptions of Theorem 4 . We then obtain, under any of these assumptions,

$$
\lim _{t \rightarrow \infty} t \mathrm{P}\left(X_{i} Y_{i}>\gamma(t)\right)=\mu\left(T_{i}\right)
$$

This gives the numerator announced in (25). The denominator is obtained by applying Lemma 3 twice. Indeed, from (26), for any $i, j \in\{1, \ldots, d\}$,

$$
\lim _{p \rightarrow 1} \frac{\operatorname{VaR}_{p}\left(X_{j} Y_{j}\right)}{\operatorname{VaR}_{p}\left(X_{i} Y_{i}\right)}=\left\{\frac{\mu\left(T_{j}\right)}{\mu\left(T_{i}\right)}\right\}^{1 / \alpha},
$$

so that, for any $i \in\{1, \ldots, d\}$,

$$
\lim _{p \rightarrow 1} \frac{\operatorname{VaR}_{p}(Z)}{\operatorname{VaR}_{p}\left(X_{i} Y_{i}\right)}=\lim _{p \rightarrow 0} \sum_{j=1}^{d} \frac{\operatorname{VaR}_{p}\left(X_{j} Y_{j}\right)}{\operatorname{VaR}_{p}\left(X_{i} Y_{i}\right)}=\sum_{j=1}^{d}\left\{\frac{\mu\left(T_{j}\right)}{\mu\left(T_{i}\right)}\right\}^{1 / \alpha} .
$$

This is still equivalent, thanks again to Lemma 3, to

$$
\lim _{t \rightarrow \infty} \frac{\mathrm{P}(Z>\gamma(t))}{\mathrm{P}\left(X_{i} Y_{i}>\gamma(t)\right)}=\left[\sum_{j=1}^{d}\left\{\frac{\mu\left(T_{j}\right)}{\mu\left(T_{i}\right)}\right\}^{1 / \alpha}\right]^{\alpha} .
$$

Combining the last limit with (26) yields

$$
\lim _{t \rightarrow \infty} t \mathrm{P}(Z>\gamma(t))=\left[\sum_{j=1}^{d}\left\{\mu\left(T_{j}\right)\right\}^{1 / \alpha}\right]^{\alpha},
$$

which is the expected denominator in (25). Checking that $\mu\left(T_{j}\right)$ has the different expressions announced under the assumptions of Theorems 1-4 is straightforward, completing the proof of Corollary 5. 


\section{Acknowledgements}

This research was supported in part by the Agence Nationale de la Recherche through the AST\&RISK project (ANR-08-BLAN-0314-01). The authors are grateful to Stéphane Loisel who gave several constructive comments towards the final version of the paper.

\section{References}

Alink, S., Löwe, M. AND Wüthrich, M. V. (2004). Diversification of aggregate dependent risks. Insurance Math. Econom. 35, 77-95.

Alink, S., Löwe, M. AND WÜthrich, M. V. (2005). Analysis of the expected shortfall of aggregate dependent risks. ASTIN Bull. 35, 25-43.

Arbenz, P., Embrechts, P. And Puccetti, G. (2011). The AEP algorithm for the fast computation of the distribution of the sum of dependent random variables. Bernoulli 17, 562-591.

Barbe, P., Fougères, A.-L. And Genest, C. (2006). On the tail behaviour of sums of dependent risks. ASTIN Bull. 36, 361-373.

Basrak, B., Davis, R. A. And Mikosch, T. (2002). Regular variation of GARCH processes. Stoch. Process. Appl. 99, 95-115.

Beirlant, J., Goegebeur, Y., Teugels, J. And Segers, J. (2004). Statistics of Extremes. John Wiley, Chichester.

CAI, J. (2002). Ruin probabilities with dependent rates of interest. J. Appl. Prob. 39, 312-323.

Chen, Y. AND Su, C. (2006). Finite time ruin probability with heavy-tailed insurance and financial risks. Statist. Prob. Lett. 76, 1812-1820.

Cossette, H. And Marceau, E. (2000). The discrete-time risk model with correlated classes of business. Insurance Math. Econom. 26, 133-149.

Davis, R. A. AND Mikosch, T. (2001). Point process convergence of stochastic volatility processes with application to sample autocorrelation. In Probability, Statistics and Seismology (J. Appl. Prob. Spec. Vol. 38A), ed. D. J. Daley, Applied Probability Trust, Sheffield, pp. 93-104.

De HaAn, L. AND RESNick, S. I. (1977). Limit theory for multivariate sample extremes. Z. Wahrscheinlichkeitsth. 40, 317-337.

Degen, M., LAmbrigger, D. AND Segers, J. (2010). Risk concentration and diversification: second-order properties. Insurance Math. Econom. 46, 541-546.

Embrechts, P. And Veraverbeke, N. (1982). Estimates for the probability of ruin with special emphasis on the possibility of large claims. Insurance Math. Econom. 1, 55-72.

Embrechts, P., KlüPPelberg, C. And Mikosch, T. (1997). Modelling Extremal Events (Appl. Math. 33). Springer, Berlin.

Embrechts, P., Lambrigger, D. D. And Wüthrich, M. V. (2009a). Multivariate extremes and the aggregation of dependent risks: examples and counter-examples. Extremes 12, 107-127.

Embrechts, P., Nešlehová, J. AND WÜthrich, M. V. (2009b). Additivity properties for value-at-risk under Archimedean dependence and heavy-tailedness. Insurance Math. Econom. 44, 164-169.

Foss, S. AND Richards, A. (2010). On sums of conditionally independent subexponential random variables. Math. Operat. Res. 35, 102-119.

Goovaerts, M. J. et al. (2005). The tail probability of discounted sums of Pareto-like losses in insurance. Scand. Actuarial J. 2005, 446-461.

Hult, H. AND SAmorodnitsky, G. (2008). Tail probabilities for infinite series of regularly varying random vectors. Bernoulli 14, 838-864.

Kortschak, D. ANd Albrecher, H. (2009). Asymptotic results for the sum of dependent non-identically distributed random variables. Methodology Comput. Appl. Prob. 11, 279-306.

MAINIK, G. AND RÜSCHENDORF, L. (2009). On optimal portfolio diversification with respect to extreme risks. Preprint.

Maulik, K., Resnick, S. And Rootzén, H. (2002). Asymptotic independence and a network traffic model. J. Appl. Prob. 39, 671-699.

Nelson, D. B. (1991). Conditional heteroskedasticity in asset returns: a new approach. Econometrica 59, 347-370.

NG, K. W., TANG, Q. H. AND YANG, H. (2002). Maxima of sums of heavy-tailed random variables. ASTIN Bull. 32, 43-55.

Nyrhinen, H. (1999). On the ruin probabilities in a general economic environment. Stoch. Process. Appl. 83, 319-330.

Resnick, S. I. (1987). Extreme Values, Regular Variation, and Point Processes. Springer, New York.

ReSNICK, S. I. (2007). Heavy-Tail Phenomena. Springer, New York.

Sgibnev, M. S. (1996). On the distribution of the maxima of partial sums. Statist. Prob. Lett. 28, 235-238.

TANG, Q. AND Tsitsiashvili, G. (2003a). Precise estimates for the ruin probability in finite horizon in a discrete-time model with heavy-tailed insurance and financial risks. Stoch. Process. Appl. 108, 299-325. 
TAng, Q. AND Tsitsiashvili, G. (2003b). Randomly weighted sums of subexponential random variables with application to ruin theory. Extremes 6, 171-188.

WANG, D. AND TANG, Q. (2006). Tail probabilities of randomly weighted sums of random variables with dominated variation. Stoch. Models 22, 253-272.

WANG, D., Su, C. AND ZENG, Y. (2005). Uniform estimate for maximum of randomly weighted sums with applications to insurance risk theory. Sci. China Ser. A, 48, 1379-1394.

WÜTHRICH, M. V. (2003). Asymptotic value-at-risk estimates for sums of dependent random variables. ASTIN Bull. 33, 75-92.

Zhang, Y., Shen, X. And Weng, C. (2009). Approximation of the tail probability of randomly weighted sums and applications. Stoch. Processes Appl. 119, 655-675.

ZHu, C.-H. AND GAO, Q.-B. (2008). The uniform approximation of the tail probability of the randomly weighted sums of subexponential random variables. Statist. Prob. Lett. 78, 2552-2558. 